\title{
Systematic review examining differences in HIV, sexually transmitted infections and health-related harms between migrant and non-migrant female sex workers
}

\author{
Lucy Platt, ${ }^{1}$ Pippa Grenfell, ${ }^{1}$ Adam Fletcher, ${ }^{1}$ Annik Sorhaindo, ${ }^{2}$ Emma Jolley, ${ }_{1}$ \\ Tim Rhodes, ${ }^{1}$ Chris Bonell ${ }^{3}$
}

- Additional supplementary files are published online only. To view these files please visit the journal online (http://dx. doi.org/10.1136/sextrans2012-050491)

${ }^{1}$ Department of Social and Environmental Health Research London School of Hygiene and Tropical Medicine, London, UK ${ }^{2}$ Population Council, Mexico City, Mexico

${ }^{3}$ Department of Social Policy and Intervention, Centre for Evidence Based Intervention, University of Oxford, Oxford, UK

Correspondence to Dr Lucy Platt, Department of Social and Environmental Health Research, London School of Hygiene and Tropical Medicine, 15-17 Tavistock Place, London WC1H 9SH, UK. lucy.platt@|shtm.ac.uk.

Accepted 3 September 2012 Published Online First 30 October 2012
To cite: Platt $L$, Grenfell $P$, Fletcher $A$, et al. Sex Transm Infect 2013;89:311-319.

\section{ABSTRACT \\ Objectives To assess the evidence of differences in the risk of HIV, sexually transmitted infections (STI) and health-related behaviours between migrant and non- migrant female sex workers (FSWs).}

Methods Systematic review of published peer-reviewed articles that reported data on HIV, STIs or health-related harms among migrant compared with non-migrant FSWs. Studies were mapped to describe their methods and focus, with a narrative synthesis undertaken to describe the differences in outcomes by migration status overall and stratified by country of origin. Unadjusted ORs are presented graphically to describe differences in HIV and acute STIs among FSWs by migration and income of destination country.

Results In general, migrant FSWs working in lowerincome countries are more at risk of HIV than nonmigrants, but migrants working in higher-income countries are at less risk. HIV prevalence was higher among migrant FSWs from Africa in high-income countries. Migrant FSWs in all countries are at an increased risk of acute STIs. Study designs, definitions of FSWs and recruitment methods are diverse. Behavioural data focussed on sexual risks.

Discussion The lack of consistent differences in risk between migrants and non-migrants highlights the importance of the local context in mediating risk among migrant FSWs. The higher prevalence of HIV among some FSWs originating from African countries is likely to be due to infection at home where HIV prevalence is high. There is a need for ongoing monitoring and research to understand the nature of risk among migrants, how it differs from that of local FSWs and changes over time to inform the delivery of services.

\section{INTRODUCTION}

Migration is a key defining characteristic of the 21 st century. $^{1}$ Approximately $3.1 \%$ of the global population-or one out of every 33 persons-are migrants. Reasons for migration are multiple and complex, and can include labour, family reunification, health, security and economic disparities between high-income and low income countries. ${ }^{2}$ It is projected that during the course of this century, movement of people will become even more significant due to demographic changes as well as economic liberalisation and decline. ${ }^{1}$ Just under half (49\%) of migrants are women. ${ }^{3}$ Income opportunities for migrant women are often limited to the informal sector such as child care, domestic work or sex work, as language or legal barriers may prevent work in the formal economy. ${ }^{4}$ Increasing numbers of migrant women globally are thought to be involved in sex work. ${ }^{5-8}$

Migrants experience increased health risks and inequities in health service access, ${ }^{9}$ and this may be particularly acute among migrant women. ${ }^{10}$ Evidence has shown that women in sex work may face abuse and exploitation, ${ }^{11} 12$ and studies of migrant sex workers suggest this may be exacerbated by insecure immigration status, ${ }^{13}$ ethnic discrimination, ${ }^{14}$ ghettoised work conditions, ${ }^{15}$ reduced access to medical and legal assistance ${ }^{10} 16$ and higher rates of sexually transmitted infections (STIs). ${ }^{5}$ Research also suggests that migrants are more vulnerable to mental health problems ${ }^{1}$ and this may be particularly acute among female sex workers (FSWs). ${ }^{17}$ Research also highlights differences in risk faced by migrants according to whether they are migrating internationally or internally. International migrants travel further, incur greater financial costs migrating, have greater socio-cultural adjustments to make and are more likely to face problems regarding their legal status and access to health and other welfare services. ${ }^{18}$

Emerging evidence suggests that the size of the FSW population is correlated with countrywide prevalence of $\mathrm{HIV}^{19}$ Evidence also shows that population mobility is an important determinant in the global epidemiology of HIV and STIs, ${ }^{20}$ yet little is known about the role of migrant FSWs in the transmission of HIV and STIs. It is unclear whether migrant FSWs experience worse health outcomes and/or engage in more high-risk behaviours than non-migrant FSWs, or whether this differs by type of migration, income of destination or originating country. In the absence of a systematically reviewed and synthesised evidence base directly comparing risk between migrant and non-migrant FSWs, we undertook a systematic review to examine the differences in risk regarding health-related outcomes and risk behaviours. The objectives of the review were threefold: (1) to assess the scope and nature of research examining the effect of migration on adverse health outcomes and health-related risk among FSWs; (2) to assess whether HIV, STIs and health harms vary among FSWs according to migrant status; and (3) to assess whether this variation in HIV and STIs differ according to: (a) country of origin; and (b) income level of the country of destination. 


\section{METHODS}

The following databases were searched: MEDLINE (19502008), Embase, Social Science Citation Index, Popline, CINAHL, Global Health, African Healthline, Index Medicus for the Eastern European Region, Latin American and Caribbean Centre on Health Sciences Information, Index Medicus of the South-East Asian Region and Western Pacific Region of the Index Medicus using a search combining terms for 'sex work' and 'migrant', drawing on thesaurus and nonthesaurus terms as appropriate. We included reports written in English published from 1990 onwards that reported rates among migrant FSWs compared with non-migrant FSWs of any of the following: HIV; acute STIs (defined as current infection with syphilis, gonorrhoea or chlamydia) and other risk behaviours and health harms. A migrant was defined as someone who had travelled either internally within a country or across an international border. A sex worker was defined as someone who had ever exchanged sex for money, drugs or other goods. The review followed guidelines for systematic reviews of observational studies. ${ }^{21}$

Studies were mapped, describing their quality and focus in terms of geography, outcomes and form of migration. We undertook a narrative synthesis and described differences in prevalence of HIV and STIs by migration status and country of origin, as well as the income level of the destination country. The prevalence of HIV and STIs were stratified to observe variation by country of origin. We present unadjusted ORs of HIV, as well as STIs among migrant groups compared to non-migrant groups in order to describe variation in risk by income of the destination country. The definition of income was assigned according to 2008 gross national income per capita, calculated using the World Bank Atlas method. ${ }^{22}$ We focus on unadjusted associations because we judged that adjustment for socioeconomic status is inappropriate, as these factors may lie on the causal pathway between migration, sex work and risk, and thus potentially mediate any associations with our outcomes, and potential confounders would not be measured or controlled for consistently. ${ }^{23}{ }^{24}$ All analyses used Stata 12 (Stata Corp, College Station, Texas, USA). A full description of the methods and findings on behavioural outcomes are summarised in the web appendix.

\section{RESULTS}

\section{Methodological characteristics and focus}

Data were extracted from 26 papers that met the inclusion criteria (see web appendix). All papers that reported the results of cross-sectional analyses, although three included longitudinal data. ${ }^{25-27}$ In total, nine studies were conducted in Europe ${ }^{28-36}$ two in Australia, ${ }^{23} 37$ seven in Southeast Asia, ${ }^{38-43}$ five in sub-Saharan Africa ${ }^{25-27} 4445$ and four in Central and South America. $^{24} 46-48$ Overall, less than half of the studies $(11 / 26)$ were designed explicitly to explore the effect of migration on the outcome (table 1). ${ }^{23} 2430333537384246-48$

All studies except one $e^{32}$ described the countries of origin and destination of their migrant sample. Six studies recruited internal migrants (within their country of origin, but working and living away from their home town). ${ }^{24} 2638394144$ These studies were conducted in low-income (Ethiopia) or middle-income countries (Cameroon, China, Mexico and Thailand). Three studies included intracontinental migrants who had migrated from low-income countries to other low-income countries: Benin, ${ }^{25}$ Kenya ${ }^{27}$ and Mali. ${ }^{45}$ Three studies recruited intracontinental migrants moving from low-income to middle-income countries (Bolivia, Ecuador, Argentina, Uruguay $^{46}{ }^{47}$ and Mexico. ${ }^{48}$ ) The remaining 13 studies focussed on international or intracontinental migration moving from lowincome or middle-income to high-income countries. Destination countries included Australia, ${ }^{23} 37$ Spain, ${ }^{29}{ }^{30}$ Portugal, $^{31}$ Italy, ${ }^{34}{ }^{35}$ the Netherlands, ${ }^{36}$ UK, ${ }^{33}$ Hong Kong ${ }^{42}{ }^{43}$ and Turkey. ${ }^{28}$

Type of migration was closely linked to the income of the destination country. All intercontinental migrants moved to highincome countries. $^{30-37}$ The majority of intracontinental migrants moved to low-income $e^{25} 2745$ or lower-middle income $^{41} \quad 46$ and upper-middle income countries. ${ }^{46-48}$ The majority of internal migrants worked in lower-middle income countries, ${ }^{26} 3940$ with three studies of internal migrants in upper-middle income, ${ }^{24}$ high-income ${ }^{42}$ and low-income countries. ${ }^{44}$

Seven studies recruited participants from specialist clinics for FSWs providing STI services, ${ }^{23} 25333742-44$ eight through HIV/STI testing services, ${ }^{26-30} 323435$ five from sex work locations $^{38} 45-48$ and 10 from a combination of workplaces and clinics. $^{24} 313639-41$

Just over a third of studies did not give a definition of sex workers (11/26). Some studies specified eligibility criteria to include women who self-identified as FSWs ${ }^{23} 3238$ or used a standardised Joint United Nations Programme on HIV/AIDS definition. ${ }^{46} 474950$ Three studies relied on the staff of STI clinics, ${ }^{44}$ bar managers, health service providers ${ }^{48}$ or other FSWs to identify participants. ${ }^{26}$ One study differentiated between FSWs who injected drugs and those who did not. ${ }^{35}$ Time frames defining recent sex work varied from 4 weeks, ${ }^{33}$ 2 months and ${ }^{32} 6$ months $^{36}$ to 1 year. ${ }^{48}$

Sex work locations were diverse and 10 studies do not specify the place of sex work. ${ }^{25}$ 27-30 3435374648 Only one study involved a total sample of fewer than 100 participants, ${ }^{31}$ and five studies reported findings where either the migrant or the non-migrant sample was considerably smaller than their comparison group. ${ }^{25} 27394142$

\section{Outcomes}

Nineteen studies examined HIV as an outcome (table 2). ${ }^{24-}$ 27 29-32 34-37 39-41 44-48 Overall, prevalence was not consistently higher among migrants compared to non-migrants. Six studies examined acute STIs, ${ }^{24} 2537384851$ reporting 23 outcomes across five countries. Prevalence of an acute STI was consistently higher among migrants compared to non-migrants. The most frequently measured risk behaviour was condom use. $^{23} 2533 \quad 3742434647$ Other outcomes relating to sexual behaviours included: practising of anal sex with clients ${ }^{47}$ and accepting of extra money for unprotected sex. ${ }^{47}$ Three studies measured broader sexual and reproductive health-related outcomes, including vaginal douching with an over-the-counter medication, ${ }^{42}{ }^{43}$ undergoing a cervical smear test, ${ }^{42}{ }^{43}$ termination and use of contraceptives. ${ }^{33}$ Four studies examined use of alcohol or illegal drugs ${ }^{33} 424647$ but just one examined experience of violence. ${ }^{33}$ Socio-structural outcomes included the location of sex work ${ }^{46} 47$ and any history of incarceration or arrest. $^{33}$

\section{Variation in HIV and STI prevalence by country of origin/ transit}

In just under half of European-based studies, the prevalence of HIV was less than $1.5 \%$ among migrants and non-migrants alike. $^{29} 303336$ In all these studies, the prevalence of injecting drug use was low. In four studies, a higher prevalence of HIV 
Table 1 Characteristics of included studies

\begin{tabular}{|c|c|c|c|c|c|c|c|c|c|c|c|}
\hline Income & Country & $\begin{array}{l}\text { Data } \\
\text { collected }\end{array}$ & Outcome & $\begin{array}{l}\text { Recruitment } \\
\text { site }\end{array}$ & Location & Definition & Migrants & Non-migrants & Age (all) & $\begin{array}{l}\text { Primary } \\
\text { aim }\end{array}$ & Reference \\
\hline \multirow[t]{12}{*}{ High } & UK & 2008-2009 & $\begin{array}{l}\text { HIV STI* } \\
\text { behavioural }\end{array}$ & $\begin{array}{l}\text { Specialist } \\
\text { sexual health } \\
\text { services, flats, } \\
\text { saunas, social } \\
\text { networks }\end{array}$ & Flats, saunas & $\begin{array}{l}\text { Exchanged sex for } \\
\text { drugs, good or } \\
\text { money in last } 4 \\
\text { weeks }\end{array}$ & 163 & 105 & 26 (IQR: 22-35) & Yes & 33 \\
\hline & Spain & 2000 & HIV & HIV/STI clinic & $\begin{array}{l}\text { No } \\
\text { information }\end{array}$ & No information & 1797 & 331 & 29.2 & Yes & 30 \\
\hline & Spain & 1989-1991 & HIV & $\begin{array}{l}\text { Collaborating } \\
\text { centre and } \\
\text { snowball } \\
\text { sample }\end{array}$ & $\begin{array}{l}\text { Brothels, bar, } \\
\text { street }\end{array}$ & $\begin{array}{l}\text { Self-identified sex } \\
\text { work for money at } \\
\text { least once in last } \\
\text { year }\end{array}$ & 404 & 663 & $29.8(14-74)$ & No & 32 \\
\hline & Spain & 2000-2001 & HIV & STI clinics & $\begin{array}{l}\text { No } \\
\text { information }\end{array}$ & No information & 2607 & 523 & $29.2(S D=7.6)$ & No & 29 \\
\hline & Italy & 1988-1994 & HIV & $\begin{array}{l}\text { HIV testing } \\
\text { clinic }\end{array}$ & $\begin{array}{l}\text { No } \\
\text { information }\end{array}$ & $\begin{array}{l}\text { Professional } \\
\text { defined as } \\
\text { non-IDU }\end{array}$ & 136 & 593 & No information & Yes & 35 \\
\hline & Italy & 1991-1995 & HIV & $\begin{array}{l}\text { STI and } \\
\text { infectious } \\
\text { disease clinic }\end{array}$ & $\begin{array}{l}\text { No } \\
\text { information }\end{array}$ & $\begin{array}{l}\text { Professional } \\
\text { defined as } \\
\text { non-IDU, but } \\
\text { includes IDUs }\end{array}$ & 176 & 223 & $\begin{array}{l}\text { Median=37 } \\
(21-55)\end{array}$ & No & 34 \\
\hline & Portugal & 2000-2001 & HIV & $\begin{array}{l}\text { Clinic and } \\
\text { street }\end{array}$ & Street & No information & 54 & 42 & No info & No & 31 \\
\hline & $\begin{array}{l}\text { The } \\
\text { Netherlands }\end{array}$ & 1991 & HIV & $\begin{array}{l}\text { STI clinics } \\
\text { brothels, hotels }\end{array}$ & Indoor & $\begin{array}{l}\text { Received money or } \\
\text { goods in exchange } \\
\text { for sex at least } \\
\text { once in past } 6 \\
\text { months }\end{array}$ & 129 & 72 & $29(S D=7)$ & No & 36 \\
\hline & Australia & 1991-1993 & $\begin{array}{l}\text { HIV STI† } \\
\text { Behavioural }\end{array}$ & $\begin{array}{l}\text { Specialist } \\
\text { sexual health } \\
\text { clinic for sex } \\
\text { workers }\end{array}$ & $\begin{array}{l}\text { No } \\
\text { information }\end{array}$ & No information & 123 & 91 & $\begin{array}{l}\text { Migrant }=25.3 \\
\text { Non-migrant }=25.5\end{array}$ & Yes & 37 \\
\hline & Australia & 1990-2005 & Behavioural & $\begin{array}{l}\text { Sexual health } \\
\text { care centre and } \\
\text { primary health } \\
\text { care centre for } \\
\text { sex workers }\end{array}$ & $\begin{array}{l}\text { Parlour, } \\
\text { private } \\
\text { arrangement, } \\
\text { escorts, } \\
\text { workers }\end{array}$ & $\begin{array}{l}\text { Self-identification } \\
\text { and assessment by } \\
\text { staff }\end{array}$ & 148 & 141 & $\begin{array}{l}16-25 \text { years } \\
\text { migrant }=46 \% \\
\text { non-migrant }=50 \%\end{array}$ & Yes & 23 \\
\hline & Hong Kong & $2004-5$ & Behavioural & $\begin{array}{l}\text { Specialist } \\
\text { sexual health } \\
\text { clinic }\end{array}$ & $\begin{array}{l}\text { Street and } \\
\text { indoor }\end{array}$ & No information & 75 & 170 & $35.8(S D=6.04)$ & No & 43 \\
\hline & Hong Kong & 2005-2007 & $\begin{array}{l}\text { Behavioural } \\
\text { STI‡ }\end{array}$ & $\begin{array}{l}\text { Specialist } \\
\text { sexual health } \\
\text { clinic }\end{array}$ & $\begin{array}{l}\text { Street and } \\
\text { indoor }\end{array}$ & No information & 406 & 97 & $26-30$ years $11 \%$ & Yes & 42 \\
\hline \multirow[t]{5}{*}{ Upper-middle } & Argentina & 2000-2002 & $\begin{array}{l}\text { HIV/STI§ } \\
\text { Behavioural }\end{array}$ & Work & $\begin{array}{l}\text { Street, bar } \\
\text { and cabaret }\end{array}$ & UNAIDS definition & 169 & 456 & $\begin{array}{l}18-24 \text { yrs } \\
\text { Mig=18.4\% } \\
\text { Non }=24.3 \%\end{array}$ & Yes & 47 \\
\hline & $\begin{array}{l}\text { Argentina, } \\
\text { Bolivia, } \\
\text { Ecuador, } \\
\text { Uruguay }\end{array}$ & 1999-2002 & $\begin{array}{l}\text { HIV, STIף } \\
\text { Behavioural }\end{array}$ & Work & $\begin{array}{l}\text { No } \\
\text { information }\end{array}$ & UNAIDS definition & 185 & 1660 & No data & Yes & 46 \\
\hline & Mexico & 1998 & HIV, STI** & Bars & $\begin{array}{l}\text { No } \\
\text { information }\end{array}$ & $\begin{array}{l}\text { Identified by bar } \\
\text { managers and } \\
\text { health authorities }\end{array}$ & 363 & 121 & 25.5 & Yes & 48 \\
\hline & Mexico & 2004-2005 & HIV STI†† & $\begin{array}{l}\text { Municipal and } \\
\text { community } \\
\text { health clinics, } \\
\text { via street } \\
\text { outreach, and } \\
\text { by referrals } \\
\text { from other } \\
\text { FSWs }\end{array}$ & $\begin{array}{l}\text { Street, clubs, } \\
\text { bars }\end{array}$ & $\begin{array}{l}\text { Sex work and } \\
\text { unprotected } \\
\text { vaginal sex with } \\
\text { client once in last } \\
2 \text { months }\end{array}$ & 370 & 101 & $60 \%(18-34)$ & Yes & 24 \\
\hline & Turkey & 1993 & $\begin{array}{l}\text { Syphilis } \\
\text { markersł‡ }\end{array}$ & STI clinic & $\begin{array}{l}\text { No } \\
\text { information }\end{array}$ & $\begin{array}{l}\text { Registered sex } \\
\text { workers and } \\
\text { women arrested } \\
\text { for not being } \\
\text { registered }\end{array}$ & 96 & 88 & No data & Unclear & 28 \\
\hline Lower-middle & Cameroon & 1995-1996 & HIV & $\begin{array}{l}\text { Outreach } \\
\text { workers in } \\
\text { community } \\
\text { referred women } \\
\text { to three clinics } \\
\text { (family }\end{array}$ & Bars, home & $\begin{array}{l}\text { Having at least } \\
\text { four different sex } \\
\text { partners per } \\
\text { month }\end{array}$ & 1453 & 808 & 25.5 & No & 26 \\
\hline
\end{tabular}


Table 1 Continued

\begin{tabular}{|c|c|c|c|c|c|c|c|c|c|c|c|}
\hline Income & Country & $\begin{array}{l}\text { Data } \\
\text { collected }\end{array}$ & Outcome & $\begin{array}{l}\text { Recruitment } \\
\text { site }\end{array}$ & Location & Definition & Migrants & Non-migrants & Age (all) & $\begin{array}{l}\text { Primary } \\
\text { aim }\end{array}$ & Reference \\
\hline & & & & $\begin{array}{l}\text { planning clinic, } \\
\text { STI and } \\
\text { community } \\
\text { clinic) }\end{array}$ & & & & & & & \\
\hline & China & 2004 & $\begin{array}{l}\text { Any STI§§ } \\
\text { Behavioural }\end{array}$ & Work & Indoors & Self-identified & 151 & 264 & $23.5(S D=5.1)$ & Yes & 38 \\
\hline & China & 1998-1999 & HIV & $\begin{array}{l}\text { Hospital and } \\
\text { via outreach to } \\
\text { working } \\
\text { locations }\end{array}$ & $\begin{array}{l}\text { Bars, hotels, } \\
\text { hair dressing } \\
\text { salons, beauty } \\
\text { parlours }\end{array}$ & No information & 135 & 831 & $25(S D=5)$ & No & 40 \\
\hline & Thailand & 1992 & HIV & $\begin{array}{l}\text { STI clinic and } \\
\text { work places }\end{array}$ & Indoors & $\begin{array}{l}\text { Brothels (direct } \\
\text { workers) bars, } \\
\text { massage parlours, } \\
\text { karaoke clubs } \\
\text { (indirect) }\end{array}$ & 51 & 672 & $\begin{array}{l}48 \%(383)<20 \\
\text { years }\end{array}$ & No & 41 \\
\hline & Thailand & 1991-1994 & HIV & $\begin{array}{l}\text { STI clinic and } \\
\text { work places }\end{array}$ & Indoors & No information & 37 & 463 & 23.6 (mean) & No & 39 \\
\hline & Kenya & 1985 & HIV & STI clinic & Missing & No information & 399 & 19 & $29.2(S D=6.5)$ & No & 27 \\
\hline \multirow[t]{3}{*}{ Low } & Ethiopia & 1998 & HIV & $\begin{array}{l}\text { STI clinic set up } \\
\text { for research }\end{array}$ & $\begin{array}{l}\text { Individual, } \\
\text { shared rooms, } \\
\text { bars, street }\end{array}$ & $\begin{array}{l}\text { Known to health } \\
\text { staff as being sex } \\
\text { workers }\end{array}$ & 290 & 82 & $57 \%<24$ years & No & 44 \\
\hline & Mali & 1995 & HIV & Work & Street & No info & 94 & 82 & $28.8(15-50)$ & No & 45 \\
\hline & Benin & $\begin{array}{l}\text { 1993, } \\
1995,1999\end{array}$ & $\begin{array}{l}\text { HIV STIףף } \\
\text { Behavioural }\end{array}$ & $\begin{array}{l}\text { Specialist STI } \\
\text { clinic for sex } \\
\text { workers }\end{array}$ & $\begin{array}{l}\text { No } \\
\text { information }\end{array}$ & No information & 461 & 130 & 28.4 & No & 25 \\
\hline
\end{tabular}

The UNAIDS definition of a female sex worker is a woman who receives money or goods in exchange for sexual services, either regularly or occasionally, and who may or may not consciously define those activities as income generating.

${ }^{*}$ Antibodies to T. pallidum, chlamydia and/or Gonorrhoea.

tChlamydia, pelvic inflammatory disease, clinical genital herpes, chronic hepatitis B virus and syphilis (all stages).

¥Syphilis, gonorrhoea and chlamydia.

$\S$ Syphilis, and hepatitis B and C.

१Treponema pallidum, gonorrhoea, chlamydia, herpes simplex virus 2 and hepatitis B.

${ }^{*}$ Treponema pallidum, gonorrhoea, chlamydia, herpes simplex virus 2 and hepatitis B.

t+Chlamydia, gonorrhoea, HIV, syphilis or any STI.

¥¥Includes past infection, early latent, late latent syphilis.

$\S \S$ Gonorrhoea, chlamydia, trichomoniasis, syphilis and genital warts.

११९Syphilis, gonorrhoea and chlamydia.

EIA, enzyme immunoassay; FSW, female sex worker(s); IDU, injecting drug user; STI, sexually transmitted infections; UNAIDS, Joint United Nations Programme on HIVIAIDS.

was observed among migrants from Sub-Saharan Africa at $8 \%$ in the Netherlands, ${ }^{36} 54 \%$ and $34 \%$ in Italy $^{34} 35$ and $5.4 \%$ in Spain $^{29}$ compared to migrants from other countries and nonmigrants. HIV prevalence was higher in both Italian studies, with a corresponding higher prevalence of injecting drug use at $31 \%$ and $39 \% .^{34} 35$ In Portugal, HIV prevalence was higher among non-migrants (21\%) than migrants from Africa (7\%), and injecting drug use was reported by $60 \%$ of the sample. ${ }^{31}$

In Southeast Asia, HIV prevalence was higher, at $30 \%$ or above in two studies (both in Thailand), ${ }^{39}{ }^{41}$ while one study suggested higher prevalence among migrants from China or internal migrants from hill tribes than non-migrants. ${ }^{41}$ Another study in Thailand suggested lower prevalence among internal migrants from the upper north province compared to nonmigrants in Chiang Rai. The sample of migrants was small $(n=37)$ relative to non-migrants. ${ }^{39}$ In South and Central American countries, HIV prevalence was lower (less than 5\%), with a higher prevalence among non-migrants in Argentina and Ecuador than intracontinental migrants, but a lower prevalence among non-migrants in Uruguay. ${ }^{46}$ In Mexico, prevalence was higher among non-migrants, at 11\%, than among internal migrant FSWs at $7 \% .^{24}$

Prevalence of active syphilis was higher among intracontinental migrants from Romania working in Turkey, at 39\%, and in Benin (table 3). ${ }^{25}{ }^{28}$ In Mexico, the prevalence of syphilis was higher among non-migrants compared to internal migrants $(18 \%$ vs $13 \%) .{ }^{24}$ Gonorrhoea was higher among international migrants in
Australia, and among intracontinental migrants in Mexico and Benin, but not among internal migrants in Mexico. ${ }^{24} 253748$ Chlamydia was considerably higher among international migrants in Australia, marginally higher among intracontinental migrants in Mexico, but no higher among migrants in Benin and internal migrants in Mexico. ${ }^{24} 253748$ In two studies of internal migrants in China and Mexico, an aggregated measure of STI was no higher in migrants than among non-migrants. ${ }^{24} 38$

\section{Variation in HIV and STI prevalence by income of destination country}

Taking a weighted average of HIV prevalence by income of the destination countries showed that overall HIV prevalence did not differ by migration in high-income or upper-middle income countries, and that there was a greater difference across lowermiddle income countries and a marked difference in lowincome countries. Taking a weighted prevalence of STIs suggested that the overall prevalence of any STI was higher among migrants than non-migrants, irrespective of country income, with the exception of chlamydia, which was higher among nonmigrants in high-income countries. These findings are presented graphically by country in figures 1 and 2 .

\section{DISCUSSION}

This systematic review suggests that migrants working in lower income countries are more at risk of HIV than non-migrants, but migrants working in higher-income countries are at less risk 
Table 2 Summary of HIV prevalence by migration status

\begin{tabular}{|c|c|c|c|c|c|c|c|c|}
\hline \multicolumn{2}{|l|}{ Destination } & \multirow[b]{2}{*}{ Country/region of origin } & \multicolumn{2}{|l|}{ Migrants } & \multicolumn{2}{|c|}{ Non-migrants } & \multirow{2}{*}{$\begin{array}{l}\text { IDU } \\
(\%)\end{array}$} & \multirow[b]{2}{*}{ Reference } \\
\hline Income level & Country & & $n /$ total & $\%$ & $n /$ total & $\%$ & & \\
\hline \multirow[t]{23}{*}{ High } & UK & Eastern Europe & $2 / 168$ & 1.2 & $1 / 105$ & 0.9 & 5 & 33 \\
\hline & Spain & $\begin{array}{l}\text { All (LA, Western Europe, Central and } \\
\text { Eastern Europe, North Africa and SSA) }\end{array}$ & $1 / 1797$ & 0.6 & $3 / 331$ & 0.4 & $\mathrm{~N} / \mathrm{A}$ & 30 \\
\hline & Spain & Not specified & $12 / 404$ & 3 & $25 / 663$ & 4.0 & 0 & 32 \\
\hline & Spain & All migrants & $193 / 2607$ & 7.4 & $9 / 523$ & 1.8 & 0 & 29 \\
\hline & & SSA & & 5.0 & & & & \\
\hline & & LA & & 0.6 & & & & \\
\hline & & Eastern Europe & & 0.4 & & & & \\
\hline & Italy & All migrants & $17 / 136$ & 12.5 & $94 / 593$ & 15.8 & 29 & 35 \\
\hline & & South America* & $3 / 107$ & 3 & & & & \\
\hline & & SSA* & $12 / 22$ & 54.5 & & & & \\
\hline & & Eastern Europe* & $2 / 7$ & 28.5 & & & & \\
\hline & Italy & All migrants & $29 / 136$ & 16 & $72 / 2233$ & 32 & 40 & 34 \\
\hline & & Europet & $6 / 18$ & 33 & & & & \\
\hline & & South Americat & $4 / 109$ & 4 & & & & \\
\hline & & Africat & $18 / 48$ & 38 & & & & \\
\hline & & Asia & $1 / 1$ & 100 & & & & \\
\hline & Portugal & Africa & $4 / 54$ & 7 & 9/42 & 21 & 60 & 31 \\
\hline & The Netherlands & All migrants & $3 / 129$ & 2 & $0 / 72$ & 0 & 0 & 36 \\
\hline & & SSA $\neq$ & $2 / 26$ & 8 & & & & \\
\hline & & LA $\ddagger$ & $1 / 79$ & 1 & & & & \\
\hline & & Other $\ddagger$ & $0 / 24$ & 0 & & & & \\
\hline & Australia & Thailand, Malaysia, China & $1 / 93$ & 1 & $0 / 121$ & 0 & $7 \S$ & 37 \\
\hline & Hong Kong & Chinese (newly arrived and illegal migrants) & $1 / 412$ & 0.2 & $0 / 97$ & 0 & $\mathrm{~N} / \mathrm{A}$ & 42 \\
\hline \multicolumn{3}{|c|}{ Weighted average for high income countries } & $262 / 5936$ & 4 & $213 / 4780$ & 4.5 & & \\
\hline \multirow[t]{7}{*}{ Upper-middle } & Mexico & All migrants & $4 / 519$ & 0.7 & $1 / 121$ & 1 & 0.8 & 48 \\
\hline & & Guatemala & $4 / 357$ & 1 & & & & \\
\hline & & El Salvador & $0 / 76$ & 0 & & & & \\
\hline & & Honduras & $0 / 86$ & 0 & & & & \\
\hline & Mexico & Internal & $26 / 370$ & 7 & $11 / 101$ & 11 & $\mathrm{~N} / \mathrm{A}$ & 24 \\
\hline & Argentina & South American countries§ & $2 / 169$ & 4 & $18 / 456$ & 1 & N/A & 47 \\
\hline & Argentina & Dominican Republic, Paraguay, Uruguay & $1 / 84$ & 1 & $8 / 211$ & 4 & $\mathrm{~N} / \mathrm{A}$ & 46 \\
\hline \multicolumn{3}{|c|}{ Weighted average for upper-middle countries } & $33 / 1142$ & 3 & $38 / 889$ & 4 & & \\
\hline \multirow[t]{13}{*}{ Lower-middle } & Bolivia & Ecuador, Brazil & $0 / 6$ & 0 & $0 / 189$ & 0 & & 46 \\
\hline & Ecuador & Columbia & $0 / 28$ & 0 & 20/1019 & 2 & & 46 \\
\hline & Uruguay & Brazil & 2/67 & 3 & $2 / 241$ & 1 & & 46 \\
\hline & Thailand & All migrants & $40 / 119$ & 34 & $134 / 672$ & 20 & N/A & 41 \\
\hline & & Hill tribes & $18 / 51$ & 35 & & & & \\
\hline & & Burmese/Chinese & $22 / 68$ & 32 & & & & \\
\hline & Thailand & Internal & $3 / 37$ & 8 & $157 / 463$ & 34 & $\mathrm{~N} / \mathrm{A}$ & 39 \\
\hline & Cameroon & Internal & $319 / 1453$ & 22 & $91 / 808$ & 11 & N/A & 26 \\
\hline & Kenya & All migrants & $250 / 399$ & 63 & 9/19 & 50 & N/A & 27 \\
\hline & & Tanzania & $229 / 358$ & 60 & & & & \\
\hline & & Uganda & $18 / 37$ & 50 & & & & \\
\hline & & Other & $3 / 4$ & 80 & & & & \\
\hline & China & Internal & $7 / 831$ & 0.8 & $6 / 135$ & 0.7 & 7 & 40 \\
\hline \multicolumn{3}{|c|}{ Weighted average for lower-middle countries } & $373 / 2541$ & 15 & $410 / 3527$ & 11 & & \\
\hline \multirow[t]{10}{*}{ Low } & Ethiopia & Internal & $235 / 290$ & 81 & $40 / 82$ & 49 & $\mathrm{~N} / \mathrm{A}$ & 44 \\
\hline & Mali & All migrants & $55 / 140$ & 39 & $24 / 36$ & 67 & $\mathrm{~N} / \mathrm{A}$ & 45 \\
\hline & & Ghana & $34 / 56$ & 61 & & & & \\
\hline & & Nigeria & $12 / 58$ & 21 & & & & \\
\hline & & Other** & $9 / 26$ & 38 & & & & \\
\hline & Benintt & All migrants & $158 / 329$ & 48 & $25 / 130$ & 19 & N/A & 25 \\
\hline & & Ghana & $74 / 128$ & 58 & & & & \\
\hline & & Nigeria & $83 / 225$ & 37 & & & & \\
\hline & & Togo & $46 / 87$ & 53 & & & & \\
\hline & & Otherł‡ & $1 / 21$ & 4 & & & & \\
\hline \multicolumn{3}{|c|}{ Weighted average for low-income countries } & $698 / 1158$ & 60 & $98 / 267$ & 37 & & \\
\hline
\end{tabular}

*Countries not specified for total tested, but among those testing HIV-positive, countries included Brazil, Columbia, Senegal, Nigeria, Ghana, Zaire and ex-Yugoslavia.

tEurope includes ex-Yugoslavia, France, Switzerland and Spain; Africa includes Nigeria, Ghana, Zaire, Senegal, Tanzania, Burkino Faso and Liberia, South America includes Brazil and Columbia; Asian countries were not specified.

¥SSA=Ghana (16) and not specified (10); Latin America=Colombia (27) Dominican Republic (28), Surinam (16) and four others (6); 12 other countries (24).

$\S$ All local, no migrants were reported to be IDUs.

qIncludes Dominican Republic, Paraguay, Peru, Uruguay and Bolivia.

${ }^{* *}$ Senegal (15), Cameroon (1), Côte d'Ivoire (1), Guinea (2), Liberia (2), Niger (1), Togo (1) and unknown (2).

t+Data taken from only the 1999 phase of study as only small samples of non-migrants were available in 1993 and 1995 ( $n=7$ and 37 respectively).

$\ddagger \neq$ Countries not specified.

IDU, Injecting drug user; LA, Latin America; SSA, Sub-Saharan Africa. 
Table 3 Summary of STI prevalence by migration status and country of origin

\begin{tabular}{|c|c|c|c|c|c|c|c|c|}
\hline \multirow[b]{2}{*}{$\begin{array}{l}\text { Income } \\
\text { level }\end{array}$} & \multirow[b]{2}{*}{ Country } & \multirow[b]{2}{*}{ STI outcome } & \multirow[b]{2}{*}{$\begin{array}{l}\text { Country of origin/migration } \\
\text { status }\end{array}$} & \multicolumn{2}{|l|}{ Migrant } & \multicolumn{2}{|l|}{ Non-migrant } & \multirow[b]{2}{*}{ Reference } \\
\hline & & & & $\begin{array}{l}\text { STI positive/ } \\
\text { total }\end{array}$ & $\%$ & $\begin{array}{l}\text { STI positive/ } \\
\text { total }\end{array}$ & $\%$ & \\
\hline \multirow[t]{10}{*}{ High } & \multirow[t]{2}{*}{ UK } & Chlamydia & \multirow[t]{2}{*}{ Eastern Europe } & $7 / 140$ & 5 & $3 / 92$ & 3 & \multirow[t]{2}{*}{33} \\
\hline & & Gonorrhoea & & $5 / 150$ & 4 & $0 / 92$ & 0 & \\
\hline & \multirow[t]{4}{*}{ Australia } & Gonorrhoea (all sites) & Thailand, Malaysia, China & $17 / 123$ & 14 & $0 / 91$ & 0 & \multirow[t]{4}{*}{37} \\
\hline & & Gonorrhoea (urogenital) & & $14 / 123$ & 11 & $0 / 91$ & 0 & \\
\hline & & Chlamydia & & $18 / 123$ & 15 & $0 / 91$ & 0 & \\
\hline & & Trichomonias & & $1 / 123$ & 1 & $0 / 91$ & 0 & \\
\hline & Hong & Gonorrhoea & Legal migrants & $3 / 361$ & 0.8 & $3 / 97$ & 3.1 & \multirow[t]{4}{*}{42} \\
\hline & \multirow[t]{3}{*}{ Kong } & & Illegal migrants & $3 / 45$ & 7 & $3 / 97$ & 3.1 & \\
\hline & & Chlamydia & Legal migrants & $12 / 361$ & 3.3 & $8 / 97$ & 8.2 & \\
\hline & & & Illegal migrants & $3 / 45$ & 7 & $8 / 97$ & 8.2 & \\
\hline \multirow{3}{*}{\multicolumn{2}{|c|}{ Weighted average }} & All STIs & & $83 / 1594$ & 5 & $25 / 742$ & 3 & \\
\hline & & Chlamydia & & $31 / 669$ & 5 & $19 / 280$ & 7 & \\
\hline & & Gonorrhoea & & $28 / 679$ & 4 & $6 / 280$ & 2 & \\
\hline \multirow[t]{14}{*}{ Upper-middle } & \multirow[t]{2}{*}{ Turkey } & $\begin{array}{l}\text { Early latent syphilis (EIA } \\
\text { reactivity) }\end{array}$ & Romania & $12 / 96$ & 13 & $7 / 88$ & 8 & \multirow[t]{2}{*}{28} \\
\hline & & All Syphilis\# (IgG.TPA) & Romania & $37 / 96$ & 39 & $15 / 88$ & 17 & \\
\hline & \multirow[t]{4}{*}{ Mexico } & Gonorrhoea & All migrants & $39 / 288$ & 14 & $14 / 212$ & 7 & \multirow[t]{4}{*}{48} \\
\hline & & & Guatemala & $24 / 162$ & 15 & $6 / 88$ & 7 & \\
\hline & & & El Salvador & $8 / 62$ & 13 & $4 / 62$ & 6 & \\
\hline & & & Honduras & $7 / 64$ & 11 & $4 / 64$ & 6 & \\
\hline & Mexico & Chlamydia & All migrants & $43 / 289$ & 15 & $29 / 226$ & 13 & 48 \\
\hline & & & Guatemala & $24 / 162$ & 15 & $13 / 99$ & 13 & \\
\hline & & & El Salvador & $10 / 63$ & 16 & $8 / 63$ & 13 & \\
\hline & & & Honduras & $9 / 64$ & 14 & $8 / 64$ & $12.5 \%$ & \\
\hline & Mexico & Syphilis* & Internal & $48 / 370$ & 13 & $18 / 101$ & 18 & 24 \\
\hline & & Chlamydia & & $59 / 370$ & 16 & $19 / 101$ & 19 & \\
\hline & & Gonorrhoea & & $30 / 370$ & 8 & $13 / 101$ & 13 & \\
\hline & & Any STI & & $115 / 370$ & 31 & $47 / 101$ & 47 & \\
\hline Weighted averag & & All STIs & & 246/1139 & 22 & $112 / 715$ & 16 & \\
\hline Lower-middle & China & Any STI infectiont & Internal & $49 / 147$ & 33 & $124 / 264$ & 47 & 38 \\
\hline Low & Benin $\ddagger$ & Syphilis§ & Ghana & $4 / 128$ & 3 & $1 / 130$ & 1 & 25 \\
\hline & & & Nigeria & $2 / 225$ & 1 & $1 / 130$ & 1 & \\
\hline & & & Togo & $3 / 87$ & 3 & $1 / 130$ & 1 & \\
\hline & & & Other & $1 / 21$ & 4 & $1 / 130$ & 1 & \\
\hline & & & All migrants & $10 / 461$ & 5 & $1 / 130$ & 1 & \\
\hline & Benin & Gonorrhoea & Ghana & $35 / 128$ & 27 & $14 / 130$ & 11 & 25 \\
\hline & & & Nigeria & $50 / 225$ & 22 & $14 / 130$ & 11 & \\
\hline & & & Togo & $22 / 87$ & 25 & $14 / 130$ & 11 & \\
\hline & & & Otherq & $1 / 21$ & 4 & $14 / 130$ & 11 & \\
\hline & & & All migrants & $108 / 461$ & 23 & $14 / 130$ & 11 & \\
\hline & Benin & Chlamydia & Ghana & $6 / 128$ & 5 & $10 / 130$ & 8 & 25 \\
\hline & & & Nigeria & $11 / 225$ & 5 & $10 / 130$ & 8 & \\
\hline & & & Togo & $3 / 87$ & 4 & $10 / 130$ & 8 & \\
\hline & & & Otherף & $1 / 21$ & 3 & $10 / 130$ & 8 & \\
\hline & & & All migrants & $21 / 461$ & 5 & $10 / 130$ & 8 & \\
\hline Weighted averag & & All STIs & & $139 / 1383$ & 10 & $25 / 390$ & 6 & \\
\hline $\begin{array}{l}\text { *Rapid plasma } \\
\text { †Testing positive } \\
\text { ‡Data are taken } \\
\text { §Active syphilis } \\
\text { १Not specified. } \\
\text { \#All syphilis incl } \\
\text { EIA, enzyme imr }\end{array}$ & $\begin{array}{l}\text { reagin titres } \\
\text { e at the tim } \\
\text { from only } \\
\text { was diagno } \\
\text { ludes past ir } \\
\text { munoassay. }\end{array}$ & $\begin{array}{l}8 \text { were considered to be } \\
\text { survey for either syphilis, } \\
999 \text { phase of study as only } \\
\text { when both the rapid plasm } \\
\text { ion, early latent and late la }\end{array}$ & $\begin{array}{l}f \text { active infection. } \\
\text { chlamydia, genital warts or tricho } \\
\text { nples of non-migrants were availab } \\
\text { nd } T \text {. pallidum haemagglutination } \\
\text { lis. }\end{array}$ & $\begin{array}{l}\text { as. } \\
1993 \text { and } 1995 \\
\text { pallidum particle }\end{array}$ & $\begin{array}{l}\text { od } 37) \\
\text { tinatic }\end{array}$ & $\begin{array}{l}\text { espectively. } \\
\text { ) tests were posi }\end{array}$ & & \\
\hline
\end{tabular}

of HIV. Migrant FSWs in both low-income and high-income countries are at a greater risk of acute STIs than non-migrants.

HIV prevalence was higher among migrant FSWs from Africa in high-income countries (where there was no injecting drug use) than among non-migrant and other migrant FSWs. This is more likely to reflect the higher prevalence in countries of origin than reflecting more risk among African migrants compared to other migrants in destination countries. It is also likely that pre-migration factors contribute to risk. And increased risk of non-acute STIs has been documented among migrants from Central and Eastern Europe, ${ }^{28} 52$ suggesting that country of origin may reflect past sexual behaviour as well as the prevalence of STIs in country of origin. Some studies suggest that HIV prevalence was lower among emigrant FSWs than among their counterparts at home..$^{23} 293641$ Multiple factors may explain this, including the lower prevalence of HIV among heterosexual men in the destination countries, better organisation of local sex work or access to sexual health services, or a tendency for the healthiest populations to migrate. ${ }^{20}$

An increased risk of acute STIs was more frequently reported among migrant FSWs than among non-migrants, supporting evidence elsewhere that migrant communities engage in higher risk 
Figure 1 Odds ratio of HIV against migrant versus non-migrant sex workers by income of destination country.

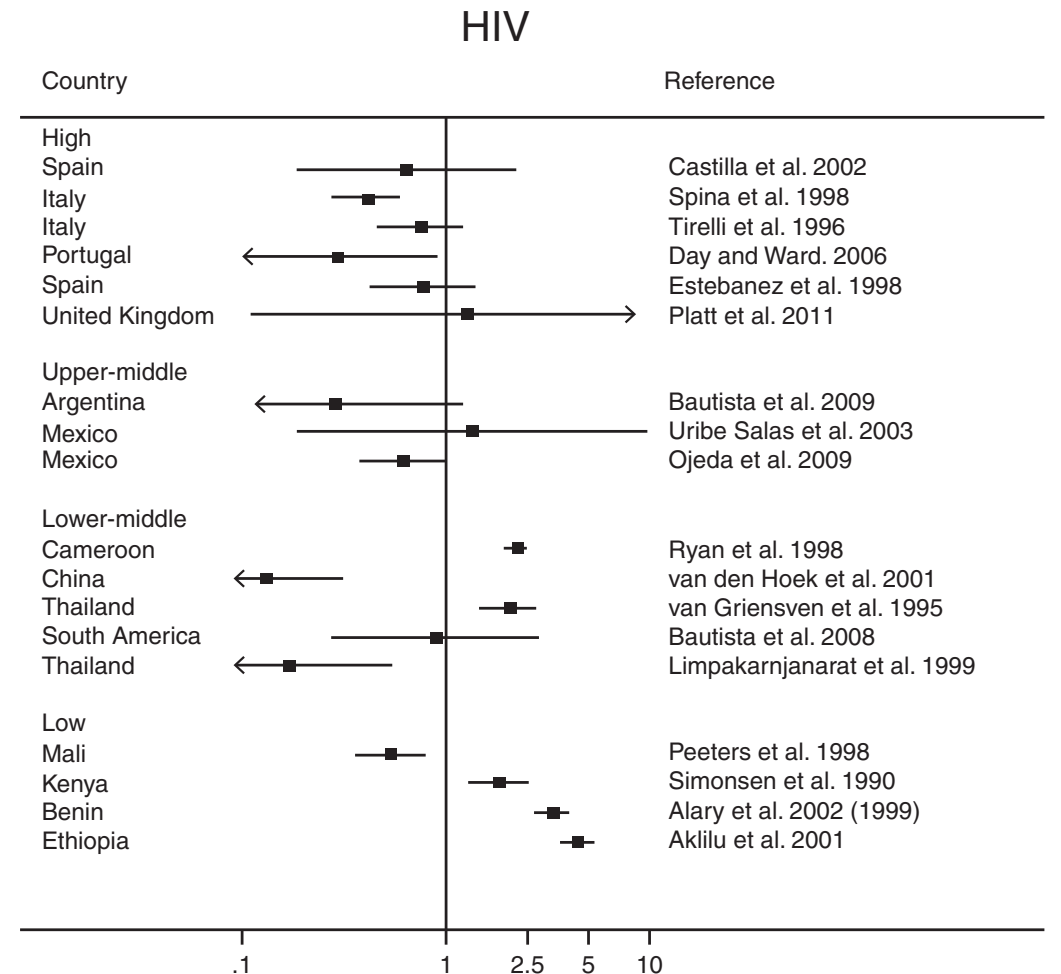

sexual activities immediately after arrival. ${ }^{10} 16$ This difference was not entirely consistent, suggesting that community-level factors shaping the local organisation of sex work in turn shape health behaviours. ${ }^{6}$ In some contexts, street sex work carries more risk than indoor venues, while the reverse has also been shown. ${ }^{1153}$ Structural policies in the country of destination are key to shaping risk behaviours among migrants. In Hong Kong, for example, migrants without residency permits had fewer options for their working locations and were subject to a fee for medical services-factors that might contribute to their higher prevalence of syphilis and gonorrhoea compared to their migrant counterparts with residency status. ${ }^{42}$ Similarly, an increased risk of STIs has been shown in Germany among FSWs without health insurance. ${ }^{54}$ Evidence points to the role of gender in shaping risk among migrant populations, as indicated by the reduced prevalence of HIV among South American FSWs migrating internally and internationally 293435 but an elevated prevalence among transgender migrants from South America working in the Netherlands and Italy. ${ }^{53} 55$ As in other studies, our findings illustrate the importance of injecting drug use in increasing the risk of HIV among FSWs ${ }^{31} 34 \quad 3553$ and higher levels of drug use among non-migrants than among migrant populations. $^{37} 46$

\section{Methodological focus and scope}

Behavioural data predominantly focussed on sexual risk behaviours, specifically condom use with clients. There was a lack of research on other harm associated with sex work and migration,
Figure 2 Odds ratio of STI against migrant versus non-migrant sex workers by income of destination country.

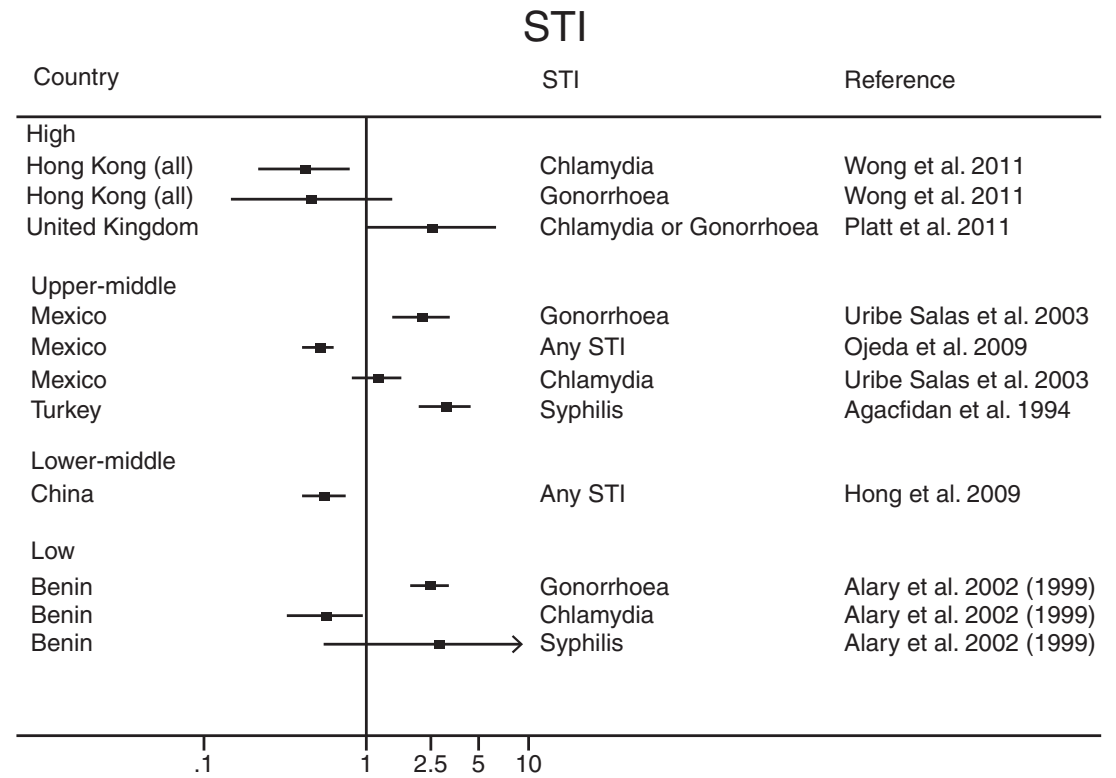


with just one study measuring violence and none addressing mental health, although these have been explored elsewhere. ${ }^{1756}$ This may, in part, result from excluding qualitative literature from the review, and therefore there is scope for a synthesis of published qualitative and mixed-methods research in this area, and for future quantitative work to be informed by existing qualitative data on broader health issues. ${ }^{57} \mathrm{~A}$ focus on sexual risk behaviours makes sense in countries with a high prevalence of HIV/STI but not in low prevalence countries where measures of sexual health should encompass the use of contraceptives, pregnancies and violence. The narrower focus on STIs may be dictated by historical approaches to health interventions that viewed FSWs as transmission bridges between high-risk and low-risk populations and emphasised the need to protect the health of the general public rather than the health needs of women. ${ }^{3}$ Our review found no studies conducted in North America, which was surprising, considering the high levels of migration and the wealth of research on risk associated with sex work in the region. ${ }^{12}$

Only a third of studies explicitly set out to examine the effect of migration on the risks associated with sex work as the primary hypothesis, with the majority examining the effect of migration as a secondary hypothesis. This may have led to an under-powering in the sample size to determine an effect, as well as lack of clarity in definitions of a migrant. ${ }^{31} 39414345$ All the studies included are observational and so fail to establish the causality of the mechanisms by which migration influences HIV risk, with the exception of one study that investigated illegal residency status as a barrier. ${ }^{42}$ Other studies of migrant workers, such as mine workers in South Africa, have established ways in which risky working conditions, a lack of social support and separation from family can lead to unprotected sex with FSWs. ${ }^{58}$ There is a need to map the causal pathways through which migration influences risk of HIV among migrant FSWs more clearly. ${ }^{59}$

\section{Limitations}

By limiting the search to literature written in English only, we may have missed key studies. It was not possible to impose a standardised definition of sex work as an inclusion criterion in the review, since the definition varied widely, and the possibility that some studies sampled women no longer engaging in sex work cannot be excluded. Similarly, inconsistency in the behavioural outcomes and the wide range of STI outcomes reported prevented any meta-analysis. In many cases, it was not possible to aggregate the STI data to create a composite measure of an STI outcome; either the overall prevalence or a measure of co-infection was not reported, limiting any comparison in risk of STIs by country of origin. A meta-analysis focussed on HIV was precluded because any analysis likely to be confounded by geographical location. Additionally, study definitions of migrancy often failed to capture the heterogeneity of migration among the groups or other aspects of mobility such as temporal or seasonal work. In respect to internal migration, we failed to accommodate differences between rural-to-urban or urban-to-urban migration or reasons for migration.

\section{Implications for research and policy}

The findings presented here focus on univariate associations of risk associated with migration status. Research which adjusts for pre-migration measures of risk such as age of sexual debut and pre-migration sex work is needed to help isolate the effect of migration on HIV, STIs and risk behaviours. Other known confounders associated with sex work and HIV such as injecting drug use and location of sex work should be considered. Only two studies adjusted for the language abilities of migrant women, ${ }^{23}$ which is an important measure of FSWs' ability to negotiate safe sex with clients and navigate health systems. ${ }^{54}$ There is a need for ongoing monitoring in risk behaviours, STIs and uptake of service by health and social services working with SWs as well as on-going qualitative and quantitative research to help understand the extent and nature of risk among migrants, how it may differ from that of local FSWs and how it changes over time. This will enable services to cater for the specific needs of the local population. In the short term, migration policies restricting migrants' use of health services need to be removed to increase access to services, alongside the provision of translated materials and interpreters to facilitate communication.

\section{Key messages}

- Migrant female sex workers (FSWs) in lower-income countries are more at risk of HIV than non-migrants and at less risk in higher-income countries. The risk of acute sexually transmitted infections is higher among migrants in all countries.

- The higher prevalence of HIV among some FSWs originating from African countries is likely to be due to infection at home, where HIV prevalence is high.

- The lack of a consistent difference in risk between migrants and non-migrants highlights the importance of the local context in mediating risk among migrant FSWs.

- There is a need for ongoing monitoring and research to understand the nature of risk among migrants, how it differs from that of local FSWs and changes over time to inform the delivery of services.

Acknowledgements The study was funded by the Medical Research Council Sexual Health and HIV Research Strategy Committee.

Contributors LP was the principal investigator and led the analysis and write-up of the paper. $L P, P G$ and $C B$ designed the review protocol. $P G$ conducted the literature search. $L P, P G, C B, A F$ and $A S$ reviewed the abstracts and extracted data. EJ updated the literature review. TR and all authors contributed to the writing and approved the report.

Funding Medical Research Council.

Competing interests None.

Provenance and peer review Not commissioned; externally peer reviewed.

Data sharing statement As this is a systematic review of published peer reviewed papers, all data presented are available through PubMed or academic libraries. A full reference list is presented.

\section{REFERENCES}

1 World Health Organisation. International migration, health and human rights. Geneva: World Health Organisation, 2003.

2 International Organisation for Migration. World migration report 2011. Communicating effectively about migration. Geneva: International Organisation for Migration, 2011.

3 Ward H, Aral SO. Globalisation, the sex industry, and health. Sex Transm Infect 2006;82:345-7.

4 Augustin L. Still challenging 'place': sex, money and agency in women's migrations. In: Escobar A, Harcourt W, eds. Women and the politics of place. Bloomfield, CT: Kumarian Press, 2005:40-46.

5 Cwikel JG, Lazer T, Press F, et al. Sexually transmissible infections among female sex workers: an international review with an emphasis on hard-to-access populations. Sex Health 2008;5:9-16. 
6 Goldenberg SM, Strathdee SA, Perez-Rosales MD, et al. Mobility and HIV in Centra America and Mexico: a critical review. J Immigr Minor Health 2011;14:46-84.

7 European Network for HIVISTI Prevention and Health Promotion among Migrant Sex Workers. Sex work In Europe: a mapping of the prostitution scene in 25 countries. Amsterdam: Tampep International, 2009.

8 Choi SY. Heterogeneous and vulnerable: the health risks facing transnational female sex workers. Sociol Health IIIn 2011:33:33-49.

9 Wolffers I, Verghis S, Marin M. Migration, human rights, and health. Lancet 2003:362:2019-20.

10 Soskolne V, Shtarkshall RA. Migration and HIV prevention programmes: linking structural factors, culture, and individual behaviour-an Israeli experience. Soc Sci Med 2002;55:1297-307.

11 Church S, Henderson M, Barnard M, et al. Violence by clients towards female prostitutes in different work setting: questionnaire survey. BMJ 2001;322:524-5.

12 Shannon K, Kerr T, Strathdee SA, et al. Prevalence and structural correlates of gender based violence among a prospective cohort of female sex workers. BMJ 2009;339:b2939

13 Loff B, Sanghera J. Distortions and difficulties in data for trafficking. Lancet 2004;363:566

14 Zimmerman C, Hossain M, Yun K, et al. Stolen smiles: the physical and psychological health consequences of women and adolescents trafficked in Europe. London: School of Hygiene and Tropical Medicine, 2006.

15 Wolffers I, van Beelen N. Public health and the human rights of sex workers. Lancet 2003;361:1981.

16 Matteelli A, Beltrame A, Carvalho AC, et al. Chlamydia trachomatis genital infection in migrant female sex workers in Italy. Int J STD AIDS 2003;14:591-5.

17 Ward H, Day SE. What happens to women who sell sex? Report of a unique occupational cohort. Sex Transm Infect 2006;82:413-17.

18 Zohry A. Interrelationships between internal and international migration in Egypt: a pilot study. Brighton: University of Sussex, 2005.

19 Talbott JR. Size matters: the number of prostitutes and the global HIV/AIDS pandemic. PLoS One 2007;2:e543.

20 Gushulak BD, Macpherson DW. The basic principles of migration health: population mobility and gaps in disease prevalence. Emerg Themes Epidemiol 2006;3:3.

21 Stroup DF, Berlin JA, Morton SC, et al. Meta-analysis of observational studies in epidemiology: a proposal for reporting. Meta-analysis of Observational Studies in Epidemiology (MOOSE) group. JAMA 2000;283:2008-12.

22 World Bank. Gross national income Atlas method. 2008. http://data.worldbank.org/ data-catalog\#Tables (Accessed 01 Mar 2011).

23 Fox J, Tideman RL, Gilmour S, et al. Sex work practices and condom use in female sex workers in Sydney. Int J STD AIDS 2006;17:319-23.

24 Ojeda VD, Strathdee SA, Lozada R, et al. Associations between migrant status and sexually transmitted infections among female sex workers in Tijuana, Mexico. Sex Transm Infect 2009;85:420-6.

25 Alary M, Mukenge-Tshibaka L, Bernier F, et al. Decline in the prevalence of HIV and sexually transmitted diseases among female sex workers in Cotonou, Benin, 19931999. AIDS 2002;16:463-70.

26 Ryan KA, Roddy RE, Zekeng L, et al. Characteristics associated with prevalent HIV infection among a cohort of sex workers in Cameroon. Sex Transm Infect 1998; $74: 131-5$

27 Simonsen JN, Plummer FA, Ngugi EN, et al. HIV infection among lower socioeconomic strata prostitutes in Nairobi. AIDS 1990;4:139-44.

28 Agacfidan A, Badur S, Gerikalmaz O. Syphilis prevalence among unregistered prostitutes in Istanbul, Turkey. Sex Transm Dis 1993;20:236-7.

29 Belza MJ. Prevalence of HIV, HTLV-I and HTLV-II among female sex workers in Spain, 2000-2001. Eur J Epidemiol 2004:19:279-82.

30 Castilla J, Sobrino P, del Amo J. HIV infection among people of foreign origin voluntarily tested in Spain. A comparison with national subjects. Sex Transm Infect 2002;78:250-4.

31 Day S, Ward H. Approaching health through the prism of stigma: research in seven European countries. In: Day HWS, ed. Sex work, mobility and health in Europe. London: Kegan Paul, 2006, 161-179.

32 Estebanez P, Zunzunegui MV, Aguilar MD, et al. A demographic and health survey of Spanish female sex workers: HIV prevalence and associated risk factors. J Biosoc Sci 1998:30:365-79.

33 Platt L, Grenfell P, Bonell C, et al. Risk of sexually transmitted infections and violence among indoor-working female sex workers in London: the effect of migration from Eastern Europe. Sex Transm Infect 2011;87:377-84.
34 Spina M, Mancuso S, Sinicco A, et al. Human immunodeficiency virus seroprevalence and condom use among female sex workers in Italy. Sex Transm Dis 1998:25:451-4

35 Tirelli U, Spina M, Mancuso S, et al. HIV seroprevalence study among 729 female prostitutes in Italy. J Acquir Immune Defic Syndr Hum Retrovirol 1996;11:208-9.

36 Van Haastrecht HJA, Fennema JSA, Coutinho RA, et al. HIV prevalence and risk behaviour among prostitutes and clients in Amsterdam: migrants at increased risk for HIV infection. Genitourin Med 1993;69:251-6.

$37 O^{\prime}$ Connor CC, Berry G, Rohrsheim R, et al. Sexual health and use of condoms among local and international sex workers in Sydney. Genitourin Med 1996;72:47-51.

38 Hong Y, Li XM, Yang HM, et al. HIV/AIDS-related sexual risks and migratory status among female sex workers in a rural Chinese county. AIDS Care 2009;21:212-20.

39 Limpakarnjanarat K, Mastro TD, Saisorn S, et al. HIV-1 and other sexually transmitted infections in a cohort of female sex workers in Chiang Rai, Thailand. Sex Transm Infect 1999;75:30-5.

40 van den Hoek A, Yuliang F, Dukers NH, et al. High prevalence of syphilis and other sexually transmitted diseases among sex workers in China: potential for fast spread of HIV. AIDS 2001;15:753-9.

41 van Griensven GJ, Limanonda B, Chongwatana N, et al. Socio-economic and demographic characteristics and HIV-1 infection among female commercial sex workers in Thailand. AIDS Care 1995;7:557-65.

42 Wong WC, Yim YL, Lynn H. Sexually transmitted infections among female sex workers in Hong Kong: the role of migration status. J Travel Med 2011;18:1-7.

43 Wong WCW, Wun YT, Chan KW, et al. Silent killer of the night: a feasibility study of an outreach well-women clinic for cervical cancer screening in female sex workers in Hong Kong. Int I Gynecol Cancer 2008;18:110-15.

44 Aklilu M, Messele T, Tsegaye A, et al. Factors associated with HIV-1 infection among sex workers of Addis Ababa, Ethiopia. AIDS 2001;15:87-96.

45 Peeters M, Koumare B, Mulanga C, et al. Genetic subtypes of HIV type 1 and HIV type 2 strains in commercial sex workers from Bamako, Mali. AIDS Res Hum Retroviruses 1998;14:51-8

46 Bautista CT, Mosquera C, Serra M, et al. Immigration status and HIV-risk related behaviors among female sex workers in South America. AIDS Behav 2008;12:195-201.

47 Bautista CT, Pando MA, Reynaga E, et al. Sexual practices, drug use behaviors, and prevalence of HIV, syphilis, hepatitis B and C, and HTLV-1/2 in immigrant and non-immigrant female sex workers in Argentina. J Immigr Minor Health 2009:11:99-104

48 Uribe-Salas F, Conde-Glez CJ, Juarez-Figueroa L, et al. Sociodemographic dynamics and sexually transmitted infections in female sex workers at the MexicanGuatemalan border. Sex Transm Dis 2003;30:266-71.

49 Pando MA, Berini C, Bibini M, et al. Prevalence of HIV and other sexually transmitted infections among female commercial sex workers in Argentina. Am J Trop Med Hyg 2006:74:233-8.

50 Joint United Nations Programme on HIV/AIDS. Sex work and HIV/AIDS. Geneva: UNAIDS, 2002

51 Agacfidan A, Chow JM, Pashazade H, et al. Screening of sex workers in Turkey for Chlamydia trachomatis. Sex Transm Dis 1997;24:573-5.

52 Fox J, Taylor GP, Day S, et al. How safe is safer sex? High levels of HSV-1 and HSV-2 in female sex workers in London. Epidemiol Infect 2006;134:1114-19.

53 van Veen MG, Gotz HM, van Leeuwen PA, et al. HIV and sexual risk behavior among commercial sex workers in the Netherlands. Arch Sex Behav 2010:39:714-23.

54 Nielsen S, Haar K, Sailer A, et al. STI rates and risk factors among female sex workers attending STI testing sites in Germany. International Society for Sexually Transmitted Disease Research; 10-13 July, Quebec, 2011.

55 Saleri N, Graifemberghi S, El Hamad I, et al. Prevalence and incidence of syphilis among South American transgender sex workers in northern Italy. Sex Transm Dis 2006;33:334-5.

56 Romans SE, Potter K, Martin J, et al. The mental and physical health of female sex workers: a comparative study. Aust N Z J Pyschiatry 2001;35:75-80

57 Sanders T. A continuum of risk? The management of health, physical and emotional risks by female sex workers. Sociol Health Illn 2004;26:557-74.

58 Campbell C. Migrancy, masculine identities and AIDS: the psychosocial context of HIV transmission on the South African gold mines. Soc Sci Med 1997;45:273-81.

59 Gupta GR, Parkhurst JO, Ogden JA, et al. Structural approaches to HIV prevention. Lancet 2008;372:764-75. 


\section{STl Systematic review examining differences in HIV, sexually transmitted infections and health-related harms between migrant and non-migrant female sex workers}

Lucy Platt, Pippa Grenfell, Adam Fletcher, et al.

Sex Transm Infect 2013 89: 311-319 originally published online October 30, 2012

doi: 10.1136/sextrans-2012-050491

Updated information and services can be found at:

http://sti.bmj.com/content/89/4/311.full.html

These include:

Data Supplement "Supplementary Data"

http://sti.bmj.com/content/suppl/2012/10/30/sextrans-2012-050491.DC1.html

References This article cites 49 articles, 12 of which can be accessed free at: http://sti.bmj.com/content/89/4/311.full.html\#ref-list-1

Article cited in:

http://sti.bmj.com/content/89/4/311.full.html\#related-urls

Email alerting Receive free email alerts when new articles cite this article. Sign up in service the box at the top right corner of the online article.

$\begin{array}{cc}\begin{array}{c}\text { Topic } \\ \text { Collections }\end{array} & \text { Articles on similar topics can be found in the } \\ & \text { Drugs: infectious diseases (2725 articles) } \\ & \text { HIV / AIDS (2141 articles) } \\ & \text { HIV infections (2141 articles) } \\ & \text { HIV/AIDS (2141 articles) } \\ & \text { Sex workers (435 articles) }\end{array}$

To request permissions go to:

http://group.bmj.com/group/rights-licensing/permissions

To order reprints go to:

http://journals.bmj.com/cgi/reprintform

To subscribe to BMJ go to:

http://group.bmj.com/subscribe/ 


\section{Notes}

To request permissions go to:

http://group.bmj.com/group/rights-licensing/permissions

To order reprints go to:

http://journals.bmj.com/cgi/reprintform

To subscribe to BMJ go to:

http://group.bmj.com/subscribe/ 